\title{
The Impact of Launching Surgery at the District Level in Niger
}

\author{
Rachid Sani · Babadi Nameoua $\cdot$ Abou Yahaya $\cdot$ \\ Idé Hassane - Roua Adamou - Renee Y. Hsia • \\ Patrick Hoekman · Amadou Sako · Abarchi Habibou
}

Published online: 5 August 2009

(c) The Author(s) 2009. This article is published with open access at Springerlink.com

\begin{abstract}
Background In 2005, the Ministry of Health in association with the Faculty of Medicine of Niamey decided to launch surgery at the district hospital (DH) level as part of the health strategy for the country. Surgical procedures were provided by general practitioners who received 12 months of training in basic surgery.

Methods Whereas the initiative was launched nationwide, we chose randomly to study the region of Dosso during a 1year time period of January 2007 to December 2007 in the three district hospitals as well as the regional hospital of Dosso.

Results During the course of 1 year, 544 patients received operations in the three DHs, of which $37.9 \%$ $(n=206)$ were emergent and $62.1 \%(n=338)$ were elective. The most common emergent interventions were cesarean sections (70\%) and uterine ruptures (7.8\%). For elective surgeries, hernia repairs comprised $80.8 \%$ of the cases. The mortality rate of emergent surgeries was 7.3 and $0 \%$ in the cases of elective surgeries. Of note, there was a large reduction in transfers to the regional hospital: 52\% compared to 2006 and $82 \%$ compared to 2005 . In $66.1 \%$ of
\end{abstract}

R. Sani $(\bowtie) \cdot$ P. Hoekman $\cdot$ A. Sako $\cdot$ A. Habibou

Department of Surgery, Faculty of Medicine,

University of Niamey, Niamey, Niger, West Africa

e-mail: sani_rachid@yahoo.fr

B. Nameoua

Regional Hospital of Dosso, Dosso, Niger, West Africa

A. Yahaya $\cdot$ I. Hassane $\cdot$ R. Adamou

District Hospital, Dosso Region, Dosso, Niger, West Africa

R. Y. Hsia

Department of Emergency Medicine, University of California, San Francisco, USA the transfers, the cases consisted of fractures, and in $10.4 \%$ of abdominal trauma and critical thoracic emergencies. Further study of this initiative has highlighted other challenges, including that of human resources, equipment maintenance, provision of consumables, and the need for continued training.

Conclusions Results from this governmental initiative to provide surgery in rural district hospitals by general practitioners are promising and encouraging. In the rural district of Dosso, there have been no deaths from elective surgery, and the number of surgical transfers to the regional hospital has drastically diminished.

\section{Introduction}

The inability to provide adequately for the surgical needs in many low-income countries has received increasing attention in the global health community [1,2]. One of the main barriers has been the shortage of surgical workforce [3], and there have been numerous efforts to provide different types of surgical training to various healthcare providers, from generalist physicians, clinical officers, and nurses [4-6].

Little literature has documented the specifics of these training initiatives in sub-Saharan Africa, and few studies have provided more outcomes-oriented evaluations of these programs. In Niger, the ability to offer emergency obstetrical and surgical care has been very limited in rural areas, where $72 \%$ of the population lives. In 2005, the Ministry of Health in association with the Faculty of Medicine of Niamey decided to launch surgery at the district hospital (DH) level as part of the health strategy for the country. Surgical procedures were provided by general practitioners (GPs) who received 12 months of training in 
basic surgery, who received a certificate designated "Capacity of District Surgery" (CDS). This study describes the implementation and effects of this training program for generalist physicians to provide surgical care in district hospitals rural areas after these physicians were deployed at the end of their training in 2006.

\section{Description of surgical training program}

Students chosen to be in the program were government physicians (generalists) who were already working in the District Hospital and had previously practiced in rural areas. Interested parties were required to take a written examination that covered both medical and surgical aspects of medicine, and the top students were chosen to participate in the program.

The training occurred in two stages as resident of surgery. A 3-month theoretical and practical training took place in the university hospitals. This was a defined curriculum designed by the Ministry of Health, covering aspects of general surgery (e.g., appendectomies, laparotomies, hernia repairs, splenectomy), trauma and orthopedics (e.g., reductions of open and closed fractures, amputations), obstetrics and gynecology (e.g., cesarean section, uterine rupture repair, dilatation, and curettage), and urology (e.g., testicular torsion, hydrocele repair). This training was then followed by 9 months of essential and practical training in the regional hospitals, requiring a certain number of each procedure under each category to be performed independently. The follow-up of the training was performed by the Faculty of Medicine, who named a coordinator to survey each site multiple times. The first two cohorts of trained practitioners consisted of 41 physicians from rural areas who graduated in 2006 and 2007.

To train ancillary staff, there was a concomitant program to train nurse anesthetists and surgical aides during a 3-year period, which began in 1995 by the University of Niamey. This was to provide enough technical support for the provision of these services.

These two programs were sponsored by the special program of the president and partners (Belgian Technical Cooperation, Italian Cooperation). The cost for the first year of the program was approximately $\$ 100,000$ USD, or $\$ 4,762$ per student.

\section{Methods}

For the purposes of studying the volume and outcomes of this initiative, the region of Dosso was randomly selected. Dosso is $139 \mathrm{~km}$ south of the capital and located in the southwest region of the country, which receives relatively more rain than the east of the country, and thus, more agricultural, with comparatively fewer desert areas. It is therefore relatively more populated than the east (with a greater population density compared with the arid desert in the northeast and central portions of the country); distances between district hospitals and referral hospitals in Dosso are shorter than those in the northeast and central portion of the country. This is important because our estimates of referral for more mortality and referrals will be more conservative due to even poorer accessibility in other regions.

This region has one regional hospital $(\mathrm{RH})$ and four district hospitals (DH), which serve a catchment area of $1,890,000$ inhabitants over a surface area of $31.000 \mathrm{~km}^{2}$. The districts areas were: Gaya (318,000 inhabitants), Doutchi (620,000 inhabitants), Loga (168,000 inhabitants), and Boboye (339,300 inhabitants). We wanted to evaluate the impact of this strategy beginning with the deployment of these GPs with a CDS certificate at the end of 2006 over three DHs (Gaya, Loga, and Doutchi) where these surgical services were provided.

We studied a 1-year period from January 2007 to December 2007 in the three DHs and the regional hospital of Dosso. We reviewed patient charts that had been listed in the operating room registers and hospitalization records in these four hospitals; we also analyzed the regional level reports of reported activities in addition to supervisor reports from the Ministry of Health and Faculty of Medicine. Furthermore, we obtained qualitative information regarding these initiatives through discussions with the operating teams, service chiefs, and responsible administrative personnel at the district hospitals. We studied the following measures: the trend in the number of emergency transfers from $\mathrm{DH}$ to $\mathrm{RH}$, the prevalence and type of surgical operations performed in DHs, morbidity and mortality rates of surgical procedures, and overall working conditions.

\section{Results}

In 2007, 544 patients received operations in the $3 \mathrm{DHs}$, of whom $37.9 \%(n=206)$ were emergent and $62.1 \%$ $(n=338)$ were elective. Table 1 illustrates this data by district hospital.

Table 1 Surgical activities in 2007 for the three district hospitals

\begin{tabular}{lccc}
\hline District hospitals & Emergencies & Planned surgery & Total \\
\hline Gaya & 79 & 296 & 375 \\
Doutchi & 98 & 32 & 130 \\
Loga & 29 & 10 & 39 \\
Total & 206 & 338 & 544 \\
\hline
\end{tabular}




\section{Emergent surgeries}

For emergent surgeries, cesarean sections were the most common surgical procedure and accounted for $70 \%$ $(n=144)$. As shown in Fig. 1, this procedure was followed by laparotomy for acute peritonitis accounting for $9.2 \%(n=19)$. The etiology of peritonitis was appendicitis in five cases and perforation of ileum secondary to typhoid fever in 14 cases. These two procedures were followed by hysterectomy for uterine rupture for $7.8 \%(n=16)$, repair of strangulated hernia for $4.4 \%(n=9)$ without bowel necrosis, and laparotomy for intestinal obstruction for $1.9 \%$ $(n=4)$ from adhesions. The "other" interventions included amputation, wound debridement, salpingectomy for ectopic pregnancy, and splenectomy. The mortality of all procedures combined amounted to $7.3 \%$ of cases $(n=15)$,

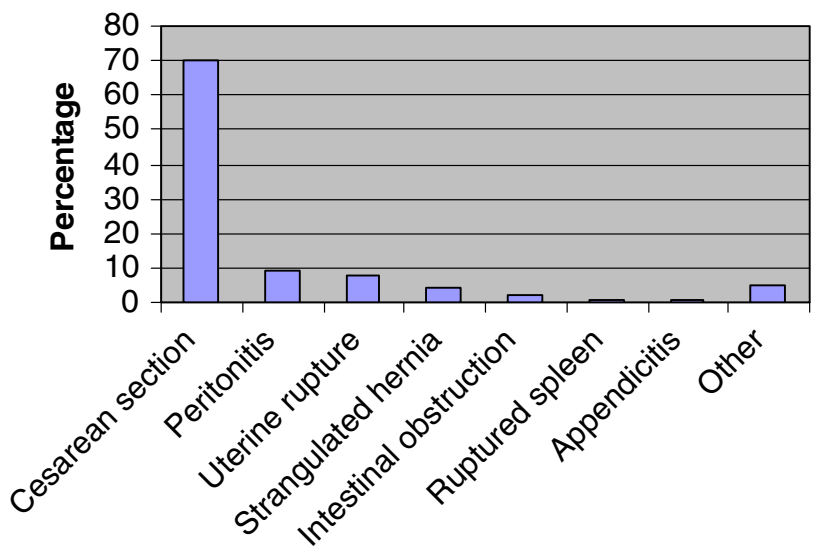

Fig. 1 Profile of the surgical emergencies treated in the three district hospitals

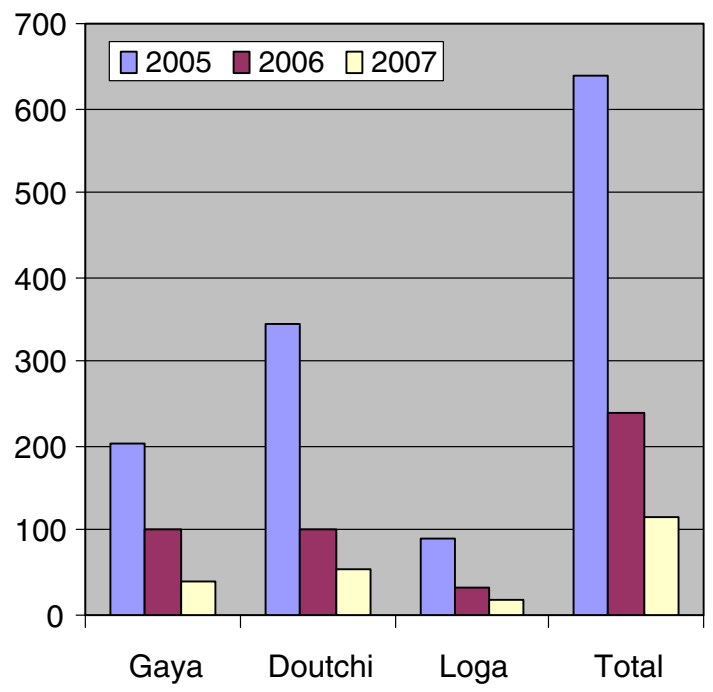

Fig. 2 Number of emergencies transferred from district hospitals to regional hospitals since the assignment of Capacity of District Surgery in district hospitals at the end of 2006
Table 2 Type of pathologies of planned surgery in the three district hospitals

\begin{tabular}{lrl}
\hline Pathology & Number & Percentage \\
\hline Hydrocele & 45 & 13.3 \\
Inguinal hernia & 218 & 64.5 \\
Umbilical hernia & 55 & 16.3 \\
Bladder stone & 4 & 1.2 \\
Lipoma & 5 & 1.5 \\
Ovarian cyst & 3 & 0.9 \\
Others (uterine fibroma, ectopic testicle, & 8 & 2.3 \\
$\quad$ uterine prolapse) & & \\
Total & 338 & 100 \\
\hline
\end{tabular}

split between 10 cases for the obstetrical procedures of cesarean section and uterine rupture $(6.25 \%$ mortality rate), and 5 cases for other interventions (10.9\% mortality rate). For comparison, the mortality rates of the regional hospital in 2007 (where surgical and obstetrical activities were performed by two general surgeons and one gynecologist) were only slightly lower: $5.7 \%$ (18/318) for obstetric and gynecologic procedures, and $9.25 \%$ for other interventions (21/227). As shown in Fig. 2, overall the rates of emergent referral to the regional hospital decreased steadily during the 3 years.

\section{Elective surgeries}

For elective cases (Table 2), hernia repair was the most performed operation (80.8\%), followed by hydrocelectomy $(13.3 \%)$. We documented 15 cases $(4.4 \%)$ of postoperative complications, the majority of which were wound infection $(66.7 \%, n=10)$. There were no cases that resulted in the death of the patient for elective procedures.

\section{Effects on referrals to regional hospital}

With respect to referrals to the regional hospital, in 2005 before the advent of surgery at the district level, there were 635 patient transferred to the regional hospital of Dosso. In 2006, some surgical activities were introduced to the district in the form of 2-week surgical camps provided by surgical teams from the National Hospital of Niamey, who came twice during the 6-month period. During this period, there were more than 240 patients transferred to a higher-level facility. In 2007, the number of transfers drastically reduced to 115 patients, which was $52 \%$ of the numbers transferred in 2006, and $82 \%$ of the numbers transferred in 2005 .

Among the 115 transferred cases, $66.1 \%(n=76)$ were fracture, $10.4 \%(n=12)$ were possible uterine rupture in high-risk patients who would require specialized postoperative treatment, $10.4 \%(n=12)$ were serious abdominal 
or thoracic trauma, and the rest $(13.1 \%$, or $n=15)$ were due to iatrogenic causes, mainly equipment malfunction (e.g., rupture of an oxygen tank or shortage of anesthetic drugs precluding further surgery).

\section{Supervision}

Supervision of these training programs in the different hospitals was led by one surgeon, one gynecologist, and one anesthesiologist-all faculty of the University of Niamey (Table 3). Each hospital was to receive six supervisory visits lasting 2 days each, although one hospital received only five due to transportation problems. Supervision included active monitoring of procedures as well as further in-service training.

After a total of 17 visits after the implementation of this district surgery program, certain problems were noted. The most common were management of human resources, in particular the poor use of specialized nurses in the operating room, and lack of technical personnel to repair and maintain surgical equipment. Providers and administrators also complained of difficulty in procuring surgical consumables (including those for orthopedic and trauma cases) and anesthetic medication. Imaging, such as ultrasound, was not available; the only imaging modality was conventional roentography or X-ray. In addition, only one operating room was functional in two of the three district hospitals. Finally, providers discussed their need for continued training in surgical techniques and management of postoperative complications.

\section{Discussion}

Reliable access to surgery for the population in rural areas has been a political preoccupation in Niger. Since 1974, mobile surgical teams had been dispatched to provide for surgical needs in these areas, but this arrangement was limited due to its sporadic and temporary nature. Niger is more than four times the size of the UK and consists mainly of desert, where the distances between health structures are significant, and poor road conditions make transfers

Table 3 Number of supervisors instituted in the district hospitals since the implementation of the district-level general practitioners trained in surgery

\begin{tabular}{llllc}
\hline Supervisors profiles & Gaya & Doutchi & Loga & Total \\
\hline RH & 3 & 3 & 3 & 9 \\
Faculty of Medicine & 2 & 2 & 1 & 5 \\
MOH & 1 & 1 & 1 & 3 \\
Total & 6 & 6 & 5 & 17
\end{tabular}

$R H$ regional hospital; $M O H$ Ministry of Health formidable and difficult. Effective coverage of surgical needs, including that of obstetrical emergencies, requires that surgical capacity be available within the area.

In Niger, the lack of surgeons is largely attributable to the prolonged training ( 5 years); the current system has only graduated three to five surgeons annually since 2004. Based on this reality, a decision was made to train general practitioners who were going to be working in rural areas in surgical skills during 12 months; In Ethiopia, the length of training consists of 6 months [7]. In Canada and Australia, in certain rural locations, surgical activities are performed by general practitioners, whereas in the United States, many procedures are provided by general surgeons [8-11].

The results of this program in Dosso since the implementation of district surgeons (or general practitioners trained in surgery) are encouraging and promising. The division of $62.1 \%$ elective and $37.9 \%$ emergent cases can be compared with the results of Humber et al. in British Columbia, Canada, of a respective split of 81 and $19 \%$ [11]. The higher percentage of emergent cases in Niger suggests that the needs for these types of programs are even greater in sub-Saharan Africa.

For elective surgeries, we found that the largest needs were in the areas of hernias and hydrocele. In fact, the mobile surgical camps that began in 1974 were specifically developed to address these pathologies in an active population (e.g., cultivators and breeders). In Niger, hernia repair is the most common gastrointestinal surgery, in nearly one of two patients [12]. The findings from a comparative study done in 2003 regarding the management of inguinal hernia at the University Hospital of Niamey and the district hospital of Gaweye showed that the pathology was treated similarly in both institutions with equivalent surgical outcomes and, in fact, the length of stay and cost of treatment was significant less in the district hospital [13]. In emergent surgeries, the obstetric interventions of cesarean and ruptured uterus represented the majority of activities (77.8\%) with a mortality rate of $6.25 \%$ and minimal morbidity, compared with $5.7 \%$ in the regional hospital where fully trained surgeons provided care. This is similar to a Canadian study where results from cesarean surgeries done by generalists was compared with that of specialists; the most surprising observation was the low morbidity rate from these major surgeries was similar for both groups. With 4-month training, on average, the generalists were practicing cesarean sections with a level of acceptable risk and safety [14].

The referrals to the regional hospital were mainly due to orthopedic trauma $(66.1 \%)$ and abdominal-thoracic trauma $(10.4 \%)$. Although these generalists are trained in the basic treatment and management of orthopedic fractures, almost half of these cases were referred to the regional hospital simply because of a lack of materials necessary for 
treatment of these orthopedic trauma cases (e.g., external fixators) and even orthopedic beds for traction. Another contributing factor to the higher referral rate for these injuries was the need for further training in these injuries.

For the management of abdominal trauma requiring splenectomy $(0.9 \%)$ at the district hospital, the transfers for serious cases required the care of a specialist and possibly further imaging, such as ultrasound. To improve the treatment of abdominal trauma, a budget would need to include the purchase of materials as well as continued education of staff in primary trauma care, as well as an additional training at the University of Niamey. Two district hospitals (Gaya, Doutchi) of the three studied in the region of Dosso are on the route of well-frequented roadways and are the main institutions that receive the patients who are victims from these road traffic injuries. According to the World Health Organization, the burden of mortality and morbidity from these types of trauma are particularly elevated in low- and middle-income countries. In fact, $90 \%$ of the burden of road traffic injuries is borne by these countries [15].

Regarding the implications of this type of training program as a method for increasing surgical workforce, the World Health Organization (WHO) has calculated that Africa bears approximately $25 \%$ of the burden of the world's diseases but only $1.3 \%$ of the world's health work force [16]. The lack of surgeons is partly related to the "brain drain" where physicians from developing countries go to more developed regions, such as Europe or the United States. The other factor contributing to the lack of surgeons is the insufficient training mechanisms, which is common across many countries in sub-Saharan Africa and has thus spawned the adoption of different political responses. In Malawi and Mozambique, for example, the health system has trained a cadre of nonphysicians called "assistant medical officers" who can perform common surgical procedures, including laparotomies and cesarean sections [16].

This particular program in Niger of equipping generalists through a dedicated surgical training is based on training physicians who have already been working in district hospitals for 2 years or more and are familiar with life in rural and more isolated areas. The other reason to choose these physicians for this type of training is the reality that all of the physicians in the 34 districts across the country are generalists. This particular training program was of interest to these physicians because this certificate of completion also provides a bonus to their annual salary, in addition to the indemnity they receive as an incentive for working in rural areas. In terms of retention, all physicians trained from this program have remained in their posts, which local officials contribute to the initial selection of trainees for this program (those already working in rural areas who originated from these areas and have settled their families there) as well as the fact that the CDS is not recognized outside of Niger.

The problem of human resources is the common denominator across all developing countries. In Niger, certain operating rooms were not functional because of lack of anesthetists, which raises the question of whether it may be necessary to train generalists in surgery and anesthesia, as in Canada [17]. According to Chen et al. [18], during the last 20 years, economic reforms have diminished public expenditure, freezing any new recruitment and weakening the salaries in the public sector. The authors also measured "health worker density" and demonstrated its correlation with survival rates, calculating that subSaharan Africa has only "a tenth of the nurses and doctors for its population" compared with Europe and, even more starkly, "Ethiopia has a fiftieth of the professionals for its population than Italy" [18]. The enormous problems must be resolved with political engagement over the short-term and long-term based on recognition of the reality that these countries face and the importance of the provision of basic life-saving surgical procedures for the population.

In Niger, the engagement of the political leaders to relieve the burden of surgical disease has been led by Ministry of Health, the University of Niamey, and development partners (e.g., the Belgian Technical Cooperation, WHO, World Bank, Italian Cooperation) to establish a short-term emphasis on the creation of the General Practitioner for District Surgery and, in the long-term, for the training of surgical specialists. The goals of these welltargeted efforts address the lack of human resources and materials. Awareness of the human resource issues in district hospitals has encouraged us to plan a strategy that, in additional to financial incentives, could enhance the satisfaction of surgeons in the practice of their profession. Their responsibility would be to follow-up outcomes from those generalists who they have trained and also provide continuous education and supervision during short stays in the district hospitals.

\section{Conclusions}

The results from this study of rural surgery performed by generalists trained in surgical procedures are promising and encouraging. Mortality and morbidity is low for both emergent and elective procedures, and referrals to the regional hospital have been reduced drastically, except for cases of fractures and abdominal-thoracic trauma. Evaluation of this program has highlighted the need for increased human resources, provision of materials, maintenance of equipment, and continuous education, especially in the field of trauma. 
Open Access This article is distributed under the terms of the Creative Commons Attribution Noncommercial License which permits any noncommercial use, distribution, and reproduction in any medium, provided the original author(s) and source are credited.

\section{References}

1. Farmer PE, Kim JY (2008) Surgery and global health: a view from beyond the OR. World J Surg 32:533-536

2. Ivers LC, Garfein ES, Augustin J et al (2008) Increasing access to surgical services for the poor in rural Haiti: surgery as a public good for public health. World J Surg 32:537-542

3. Geelhoed GW (1998) Wanted: world-class surgeons. Bull Am Coll Surg 83:32-42

4. Gruen RL (2006) Template for surgical training in resource-poor countries. ANZ J Surg 76:871-872

5. Kruk ME, Pereira C, Vaz F, Bergstrom S, Galea S (2007) Economic evaluation of surgically trained assistant medical officers in performing major obstetric surgery in Mozambique. BJOG 114:1253-1260

6. Longombe AO (1997) Surgical training of nurses for rural areas: necessity or aberration? East Cent Afr J Surg 3:43-47

7. Loutfi A, McLean AP, Pickering J (1995) Training general practitioners in surgical and obstetrical emergencies in Ethiopia. Trop Doct 25(suppl 1):22-26

8. Zuckerman R, Doty B, Gold M, Bordley J, Dietz P, Jenkins P, Heneghan S (2006) General surgery programs in small rural New York State hospitals: a pilot survey of hospital administrator. J Rural Health 22:339-342
9. Iglesias S, Saunders LD, Tracy S, Thangisalam N, Jones L (2003) Appendectomies in rural hospitals Safe whether performed by specialist or GP surgeons. Can Fam Physician 49:328-333

10. Bruening MH, Maddern GJ (1998) The provision of general surgical services in rural South Australia: a new model for rural surgery. ANZ J Surg 68:764-768

11. Humber N, Frecker T (2008) Rural surgery in British Columbia: is there anybody out there? Can J Surg 51:179-184

12. Sani R, Mc Gee JM, Illo A, Lassey JD, Madougou M, Harouna YD, Amadou M, Baoua BA, Seibou A (2004) The open tension free repair of inguinal hernia by the Lichtenstein procedure: our experience about 47 cases at the National Hospital of Niamey. J Afr Chir Dig 4:359-366

13. Sani R, Gbane M, Hennaux M, Ben Ousmane I (2007) Prise en charge des hernies inguinales simples: comparaison des aspects économiques et thérapeutiques d'un hôpital de district et l'hôpital National de Niamey-Niger. Medecine d'Afrique Noire 54:215-219

14. Aubrey-Bassler K, Newbery S, Kelly L, Weaver B, Wilson S (2007) Maternal outcomes of cesarean sections. Do generalists' patients have different outcomes than specialists' patients? Can Fam Physician 53:2132-2138

15. World Health Organization (2004) Guidelines for essential trauma care. WHO ISBN 9241546409

16. Bergström S (2005) Who will do the caesareans when there is no doctor? Finding creative solutions to the human resource crisis. BJOG 112:1168-1169

17. Chiasson PM, Roy PD (1995) Role of the general practitioner in the delivery of surgical and anesthesia services in rural western Canada. CMAJ 153:1447-1452

18. Chen L, Evans T, Anand S et al (2004) Human resources for health: overcoming the crisis. Lancet 364:1984-1990 